\title{
A Calibration Method for the Integrated Design of Finishing Robotic Workcells in the Aerospace Industry
}

\author{
Francesco Leali, Marcello Pellicciari, Fabio Pini, \\ Alberto Vergnano, and Giovanni Berselli \\ "Enzo Ferrari" Engineering Department, University of Modena and Reggio Emilia, \\ via Vignolese 905/B, 41125 Modena, Italy \\ \{francesco.leali, marcello.pellicciari, \\ fabio.pini, alberto.vergnano, giovanni.berselli\}@unimore.it
}

\begin{abstract}
Industrial robotics provides high flexibility and reconfigurability, cost effectiveness and user friendly programming for many applications but still lacks in accuracy. An effective workcell calibration reduces the errors in robotic manufacturing and contributes to extend the use of industrial robots to perform high quality finishing of complex parts in the aerospace industry. A novel workcell calibration method is embedded in an integrated design framework for an in-depth exploitation of CAD-based simulation and offline programming. The method is composed of two steps: a first offline calibration of the workpiece-independent elements in the workcell layout and a final automated online calibration of workpiece-dependent elements. The method is finally applied to a robotic workcell for finishing aluminum housings of aerospace gear transmissions, characterized by complex and non-repetitive shapes, and by severe dimensional and geometrical accuracy demands. Experimental results demonstrate enhanced performances of the robotic workcell and improved final quality of the housings.
\end{abstract}

Keywords: Workcell Calibration, Industrial Robotics, Integrated design, Aerospace industry.

\section{Introduction}

Most mechanical components in aerospace industry are characterized by complex shapes and narrow tolerance ranges, to accomplish light weight design requirements and to comply with standards and safety regulations.

The manufacturing process generally consists of two machining steps. Starting from a cast part, the first step involves the CNC machining of the functional features. In the second step the part is manually finished by removing the burrs remaining from previous machining or in zones of difficult reachability. Manual finishing requires highly skilled operators but does not assure a constant dimensional and geometric quality and it is extremely time-consuming [1].

Manufacturing robotic workcells generally provide a performing and economically sustainable alternative to manual finishing. Robotic changeable automation, in particular, has been demonstrating to be an effective solution due to its 
(re)configurability, (re)programmability and relative low cost. Its changeability, defined as the ability to cope with change or uncertainty, represents a key factor for small lot production of complex shaped parts [2].

In a changeable robotic workcell, the system is capable of heavy changes in its layout, in the settings of the mechatronic devices and in the robot's program blocks, depending on the product characteristics or on the technological process performed. The set-up time at changeover can be reduced through a modular architecture, developed following tailored design methods [3].

The authors already developed and proposed a method for designing reconfigurable robotic workcells, based on a PPR approach (Product analysis, Process identification and Resource selection). The integrated use of CAD-based environments and offline programming (OLP) tools is fundamental to satisfy the requirements on system changeability and to easily reduce the time lost for robot's reprogramming at changeover [4], [5].

Extending the application field of industrial robots from simple deburring to complex finishing and machining, the main operative limitation is given by their low precision in posing, defined in terms of resolution, repeatability and accuracy [6], [7]. The most important values used to represent precision performances of manipulators, as specified in the international standard ISO 9283, which sets the performance criteria of industrial manipulators, are pose repeatability and pose accuracy [8].

Kinematic and dynamic performances of robotic arms, in particular, depend both on mechanical and control factors and represent the major contributions to the final pose repeatability and accuracy [9].

Manufacturing and assembly tolerances on robot links introduce variations in their dimensions while the robot controller, set with nominal values, does not consider the own parameter variability of single manufactured robots.

Other typical mechanical errors, affecting the robot kinematic and dynamic behavior, are backlashes on gear and belt transmissions, friction on harmonic drives and bearings, and the intrinsic low stiffness of the robotic mechanical chain, around $1 \mathrm{~N} / \mathrm{mm}$, with respect to conventional $\mathrm{CNC}$ tool machines, with stiffness greater than $50 \mathrm{~N} / \mathrm{mm}[6]$.

Besides, the difference between the actual and the physical joint zero configuration set in the robot controller represents another importance source of uncertainties for an accurate robot pose definition.

Dynamic errors mainly depend on servo system accuracy, encoder resolution, system inertia and friction, so the robot controller is finally responsible for the trajectory deviation from its nominal definition, also due to physical loads acting on the robots (e.g. payload, gravity).

During finishing and machining, contact forces between tools and workpieces, for both part-in-hand and tool-in-hand robot configurations, influence the part quality in terms of dimensions and geometries. The tool dynamic behavior has to be investigated and machining parameters have to be carefully chosen to minimize robot's chattering and structure deformations and unsteadiness [10].

The design of auxiliary equipment is another important factor for achieving high quality in finishing and machining. Devices for tool wear control and part manipulation, for instance, need to be manufactured to assure exact reference workframes. Finally, the control of environmental factors as temperature and working 
conditions (swarf and dust collection, etc.) is very important to minimize the machining errors [11]. Fig.1 left side summarizes the main error sources affecting the robotic machining process.
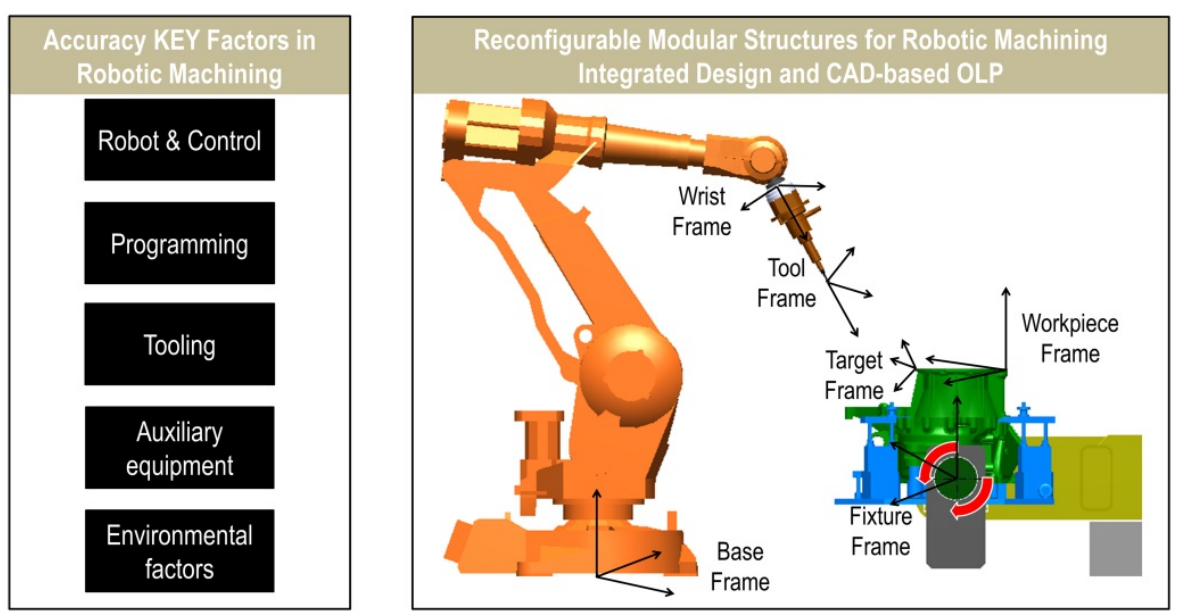

Fig. 1. Accuracy factors in robotic machining (left side) and main reference frames in a reconfigurable workcell - case with spindle on robot (right side)

In robotic finishing and machining, more than in other operations, programming and code generation represent demanding tasks. In fact the robot has to run tailored approaches with hundreds of target points toward parts subject to geometric and dimensional variability and generally inaccurately posed.

In reconfigurable workcells, moreover, the robot code has to be designed to be modular and easy to be (re)used. The robot code subprograms, workpiece dependent, have to be collected and adapted at every cycle, while non-dependent instructions have to be carefully parameterized.

Manual programming is widely diffused in robotic finishing and machining to comply with many difficulties, so that a lot of time is wasted at changeover, leading to a notable productivity loss.

OLP software, integrated by specific machining functions or dedicated packages, currently offered by several robot manufacturers and software companies for simulation and code generation, have demonstrated the capability to reduce the productivity loss at changeover [12].

The full correspondence between the real and the virtual controller implemented in the software guarantees an exact analysis of the robot's behavior and suggests a possible partial correction of the most important kinematic and dynamic errors [13].

On the other hand, the OLP approach, when applied to the design of reconfigurable robotic workcells, introduces a group of reference frames which must be used by skilled programmers, expert both in manufacturing and robot programming (for each robot brand) to modularize the robot program architecture and also define the nominal position of each element which takes part to the robotic process. Modules of robot programs and nominal position of element commonly correspond to specific reference frames, as shown in Fig.1. Anyway, misalignments between nominal and real 
positions of such elements could frustrate the advantages given by OLP in enhancing the robot's accuracy.

The present paper proposes a calibration method to bridge the gap between simulated and real robot behaviors, embedded within the design process of reconfigurable robotic workcells. The final goal is to reduce errors in robotic manufacturing and extend the use of industrial robots to perform high quality finishing of complex parts.

The next section presents a brief review of the main approaches in robot calibration proposed in the scientific literature. Section 3 describes the calibration method developed by the authors. Section 4 presents the results obtained by the application of the method for finishing complex parts in the aerospace field; the conclusions close the paper.

\section{Robotic Workcell Calibration}

According to the common terminology used in industrial robotics [14], the World Frame is the robot absolute reference. The Base Frame is the reference system which defines the robot zero position and it is located on the fixed base of the robot. In a workcell with one robot only, the Base Frame matches with the World Frame. The Wrist Frame is located on the robot wrist flange and defines the robot kinematic chain. Objects attached to the robot flange are referenced with specific frames; in case of spindle moved by the robot a Tool Frame defines the position of every tool tip. The Fixture Frame is used to identify and locate stationary or movable fixtures with respect to the Base Frame. The Workpiece Frame defines the relation between the workpiece zero point and its relative fixture, while a Target Frame is used to describe the robot's configuration at every point of a work path. The robot movements are defined with a sorted sequence of matches between the Tool Frames and the Target Frames.

As already introduced, an inaccurate definition of the reference frames has a direct impact on the final accuracy. The robot's kinematic and dynamic errors strictly depend on the correspondence between the nominal and the real dimensions of every robot link, also referred as absolute positioning inaccuracy. The misalignment from nominal to real poses of each element which takes part to the manufacturing action in the workcell layout contributes to the relative positioning error [14], [15].

The workcell calibration is the action of matching the nominal and the real pose of every reference frame which enters into the definition of the whole robotic manufacturing cycle. So a full workcell calibration considers both the singular parts of the robot (absolute calibration) and the poses of the workpieces, tools and mechatronic devices involved in finishing and machining (relative calibration) [14], [15].

Many research efforts have been spent in the past to define absolute calibration methods and instruments. The most common absolute methods are the so called model-based parametric and non-parametric calibrations [16].

Model-based calibration improves the robot accuracy through a parametric identification of the main physical error sources. Model-based calibration is defined 
by [17] and [18] along three main levels: Joint, Kinematic and Nonkinematic. The Joint Level calibration corrects the relationship between the signal produced by the transducer at every joint and its actual displacement, involving drives and joints' sensors. The Kinematic level calibration acts on the entire robot kinematic model, aiming at determining the basic kinematic geometry of the robot as well as the correct angles of every joint. The last level covers errors in robot positioning due to the dynamic effects, such as joint compliance, friction and clearance, and link compliance.

Non-parametric calibration estimates the robot's positioning errors through analytical interpolation methods which start from the measurement of the mechanical properties of the robot in predefined configurations. Absolute instruments as laser trackers or 3D cameras can be effectively employed for robot absolute calibration. Some examples can be found respectively in [13], [16], [19].

The relative positioning errors are mainly caused by dynamic changes and drift of the mechanical and/or electronic devices during the robotic operations and are difficult to correct. Other error sources are related to dimensional and geometric variations of the elements, misalignments due to the workpiece feeding and pose, tool wearing and installation of devices [15].

A further classification in relative calibration is introduced by [20], where the authors propose the distinction between calibrations which adopt external instruments and calibrations based on the use of the robot itself. Examples of external devices are coordinate measuring systems, which can be used to measure the position of the devices within the robotic workcell. This approach is particularly time consuming and has to be applied every time the workcell configuration changes, very frequent in case of small production batches. On the contrary the robot itself can be used as a carrier for accurate measuring sensors. State of the art instruments and procedures are described in [21], [22], [23].

Concluding, relative calibration highly depends on the specific robotic workcell configuration and manufacturing application so few methods are proposed as general solution for the issue. In [14] another method is presented, but it has still to be fitted into an integrated design loop involving CAD-based simulation and OLP tools.

\section{Calibration Method}

In order to deliver high quality robotic machining, an original method is presented to fully integrate the calibration approach into the design loop of reconfigurable workcells.

As previously described, calibration compares the real poses of the equipment in the robot workcell against the reference frames defined in the OLP environment. The workcell equipment can be classified in two main categories: workpiece-independent and workpiece-dependent elements. Workpiece-independent elements must be selected in function of the robotic process operations, and define the basic structure of the reconfigurable workcells. On the other hand, the workpiece-dependent elements are specific for the singular workpiece or workpiece family. Positioners, robots and 
tool racks are examples of workpiece-independent elements while workpiece fixtures and machining tools represent workpiece-dependent elements [24].

According to these two categories, the workcell calibration includes two different steps. First a layout workcell calibration is performed, defining the relative position between the robot and the workpiece-independent elements. This approach is well known in industry and allows to correct the errors share due to the mechanical inaccuracy. It is repeated just one time after the workcell assembly. Manufacturing performances obtained following this first calibration step are however not good enough to satisfy the demanding requirements of high quality finishing and machining.

Then an online calibration is realized to calibrate the reference frames defined for tools, workpiece-dependent elements and for the workpieces themselves. Such step is repeated every time a new workpiece starts its finishing or machining cycle.

The proposed calibration method uses the robot itself as a measuring machine, since the robot Wrist Frame defines the spatial position of the tool flange. To effectively exploit a robot for measuring, two extra sensors are adopted. A stationary sensor calibrates the reference frame of the robot end-effectors while a robot on-board sensor calibrates the reference frames of the elements not handled by the robot. The calibration process follows four main steps, as outlined in Fig.2.

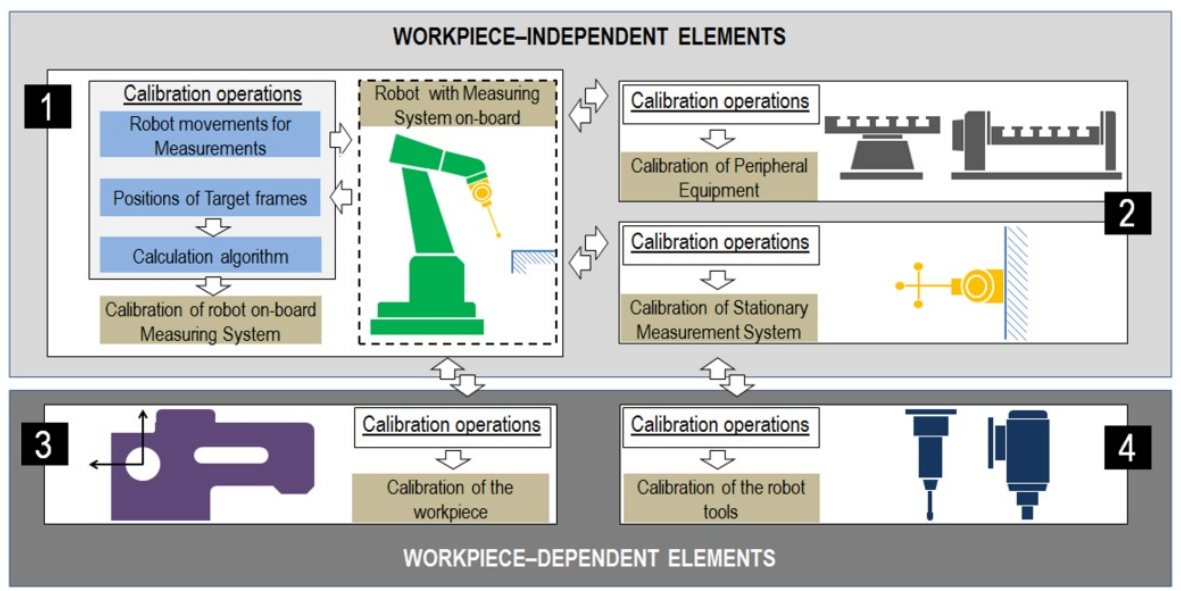

Fig. 2. Equipment and operations involved in the calibration method

The step 1 carries out the Calibration of the robot on-board measuring system. At this stage the robot is employed to define the reference system of the robot on-board sensor. The robot is moved along predefined directions with respect to a fixed accurate calibration element located in the workcell. Whenever the sensor recognizes the reference element, a target frame is self-learned by the robot. A calibration algorithm processes the recorded positions and calculates the accurate robot position relative to the on-board sensor reference frame.

The step 2 involves the robot and on-board sensor to realize the Calibration of the peripheral equipment and the Calibration of the stationary measuring system, following the same calibration process previously described. Steps 1 and 2 
accomplish the layout workcell calibration one time only, after the workcell installation.

At step 3 of the proposed method, the procedure Calibration of the workpiece defines the workpiece frame, exploiting the robot and its on-board sensor. The sensor locates the workpiece with respect to given references features, like workpiece holes, pins or planes. The Calibration of the robot tool, step 4, defines the tool frames location, and is performed using the stationary sensor located within the work volume of the robot. The last two steps conclude the workcell calibration, and are performed for each single processed workpiece.

Following the approach already proposed by [5], the method is implemented through the definition of parametric modules which simultaneously embed geometric characteristics, control logics and robot code for a quick simulation in a CAD-based offline programming environment, optimization and automation of the calibration process itself.

\section{$4 \quad$ Robotic Workcell Calibration for Improving Finishing Quality in Aerospace Industry}

The proposed method has been employed for the integrated design of a robotic workcell for accurate finishing of aerospace components. Workpieces are part of a family of aluminum housings for gear transmissions with an envelope from 4x10-3m3 to $8 \times 10-2 \mathrm{~m} 3$. Fig. 3 shows one housing and a detail before (right side, on the top) and after the robotic finishing (right side, on the bottom).
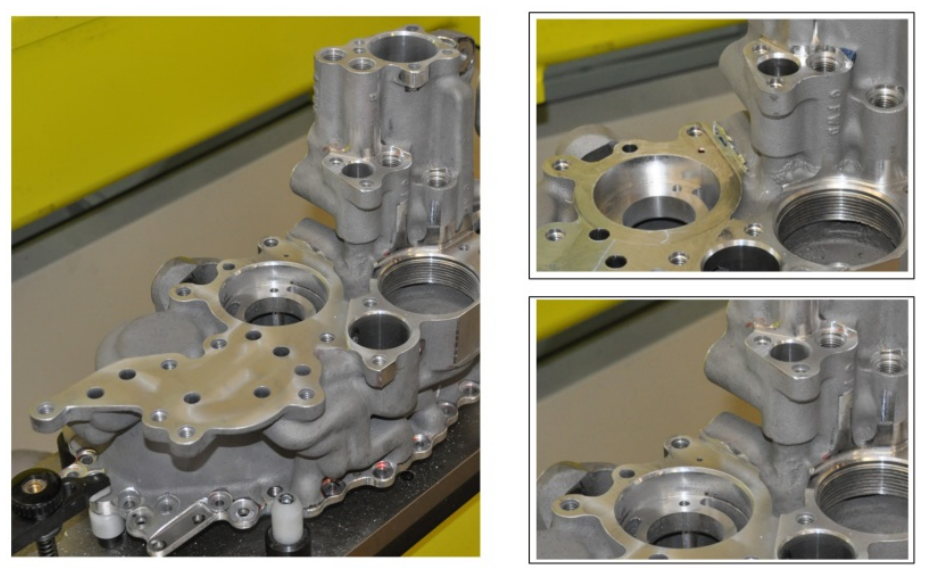

Fig. 3. Workpiece and a detail before (right top) and after finishing (right bottom)

Fig.4 shows the workcell layout. The element \#1 represents the workcell iron floor, designed for a quick transportation of the robotic workcell and to univocally fix the workpiece-independent elements. The industrial robot selected for the process, identified by \#2, is an ABB IRB 2400/16 with $16 \mathrm{~kg}$ payload and enhanced stiffness thanks to the parallelogram linkage on the third link. The workpiece feeding system at 
\#3 is an indexed positioner with two controlled tilting frames which are used to orient the workpiece during the finishing cycle. Each workpiece is locked on a mechanical interface for its quick positioning on the fixtures (element \#4). The workpiece posing and blocking are realized through a customized set of reference pins, as shown at \#5. The robot mounts a change system for a quick replacements of the end-effectors (e.g. the compliant pneumatic spindle \#6), stored in the rack \#7. The stationary sensor \#8 is a fork light barrier, located near the tool rack in order to reduce the time needed for the online calibration of the robot tools. A Renishaw RP1 inspection probe equipped with a ruby ball stylus is the on-board sensor used for both the initial calibration of the workpiece-independent elements and for the online measuring of the workpiece itself.

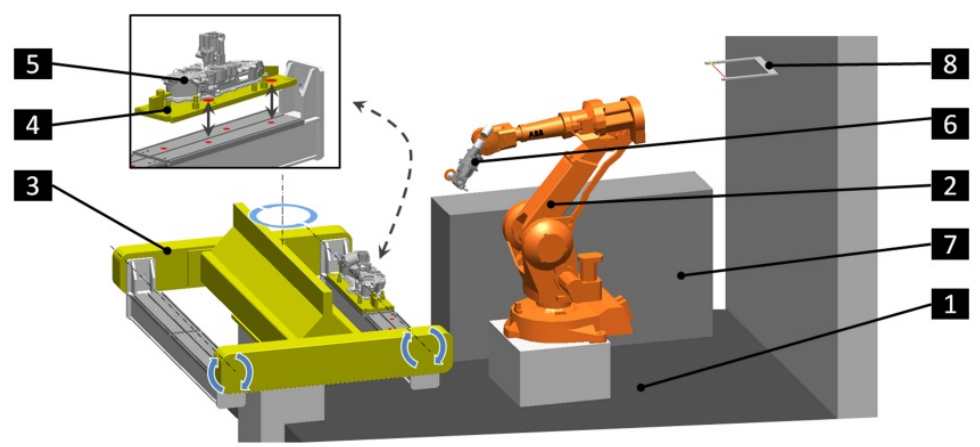

Fig. 4. Reconfigurable robotic workcell layout in ABB RobotStudio environment

ABB RobotStudio 5.14.03 is the CAD-based OLP tool adopted to implement the calibration method within the integrated design loop.

Advanced digital models of the measuring sensors have been developed through the smart functions provided by the OLP environment, for simulating, optimizing and commissioning the calibration procedure defined by the method.

A changeable and open architecture is then developed to replicate the logic behaviour of the sensors, linking independent parametric functional modules to a common skeleton, as proposed in [4].

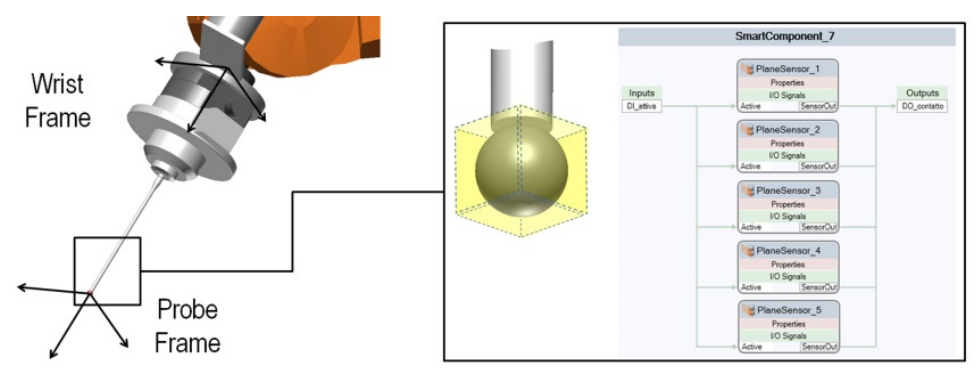

Fig. 5. The advanced model developed to replicate the logic behavior of the touch probe 
Focusing on the touch probe shown in Fig.5, five planar collision detection features replicate the probe behavior during the touching action. I/Os gates are used to communicate with the robot controller and stop the robot motors in event of collision (probe deflection). The model contains also the subprogram for robot programming which can be easily recalled within the digital environment and reused for further simulations.

The workpiece frame calibration procedure calculates the frame pose relying on measurements of a set of Target Frame at a planar surface and along two holes on the workpiece through a list of commands previously set and simulated by the user in the OLP environment. Thus, the virtual controller runs the complete calibration process, computing and updates the robot programming data type which defines the frame location, called wobjdata in the ABB RAPID native language.

Fig. 6 shows the offline simulation of the calibration process (on the left), the online calibration of the workpiece reference frame by the on-board sensor (at the center) and the online calibration of a finishing disc though the aforementioned stationary sensor (on the right), particularly important to correct the errors due to the tool wear.
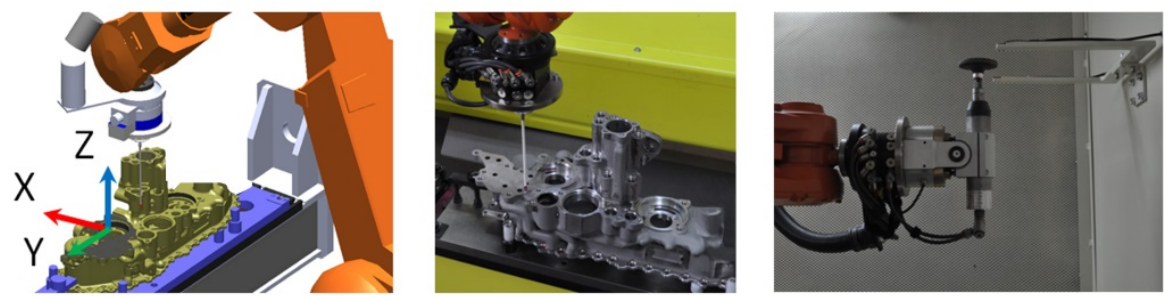

Fig. 6. Offline programming (left), online workpiece (center) and tool calibration (right)

In Fig.7 two measuring results on the workpiece borders machined with the robotic approach confirm that the border sections satisfy the quality specification. Such limit corresponds, for the application considered, to the minimum value among $0.726 \mathrm{~mm}$ and 0.3 times the length of the shortest nearest edge.
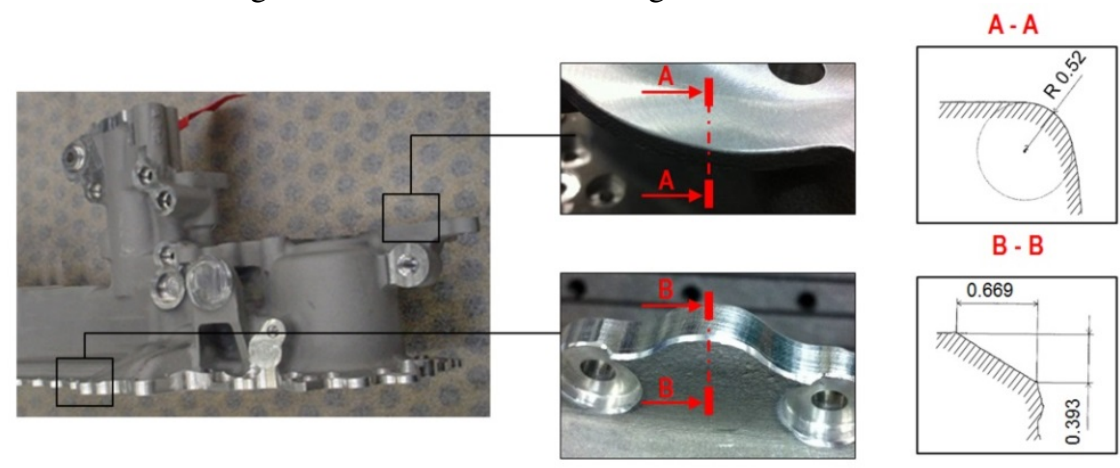

Fig. 7. Finishing of the gear transmission housing 


\section{Conclusions and Future Work}

The aerospace industry fulfills small lots production of parts that require finishing processes with high accuracy. Reconfigurable robotic systems could satisfy these requirements but need integrated design methods to enhance their modularity, both for the mechatronic devices and robot programs. Nevertheless, industrial robots deliver insufficient kinematics and dynamics accuracies for finishing operations, so it is essential to correct the different error sources.

The proposed approach leads to the development of a virtual prototype where the main features are referred to reference frames linked to every modular workcell element. The reference adjustment to match real and nominal positions can be realized with respect to the robot (absolute calibration) or to the relative positions between the workcell elements (relative calibration).

The article proposes a new calibration method consisting of two steps. First the robot handles a measuring touch probe and records every workcell element positions with respect to the spatial World Frame. This approach is already usually executed in industrial workcells just after their installation.

In the second step the robot, equipped by an on-board sensor, online measures the workpiece reference frame and the workpiece-dependent elements while a stationary sensor is used for tool reference frame online calibration.

The major novelty is that the method fully integrates the calibration phase within the engineering design cycle, i.e. finishing strategy validation, process simulation, offline programming and robot path generation, robot code commissioning. This is possible since the virtual prototype integrates the dimensional and geometric information of the used devices, the control logic necessary for the interaction with the mechatronic systems and the robot code.

The offline programming of the finishing paths and the following online calibration provide the accuracy required for the workpiece finishing, i.e. the breakage of machined to machined intersections. The final machining accuracy is a sum of various contributions; pose repeatability and accuracy, for instance, for an ABB IRB $2400 / 16$ is $\pm 0.03 \mathrm{~mm}[25]$.

Calibration does not impact on all the values of the error chain. Nevertheless the application of the calibration method significantly enhance the final quality on the workpieces. Fig.8 shows the results obtained by a human operator, commonly included between $\pm 0.1 \mathrm{~mm}$ and $\pm 0.05 \mathrm{~mm}$ when performed by a skilled workman. It is worth to compare their quality with respect to the quality achieved by the robotic arm, as shown in Fig.7.

The developed method is actually applied in programming three robotic cells for high quality finishing and machining of aerospace parts, since it makes the calibration phase simpler and more effective, improving at the same time the final quality and accuracy in finishing operations and reducing the loss productivity with respect to the manual approach.

Starting from the results presented in the paper, ongoing experiments realized in collaboration with manufacturing companies are measuring the advantages given by the method proposed also in comparison to some of the most diffused calibration methods adopted in Industry. 


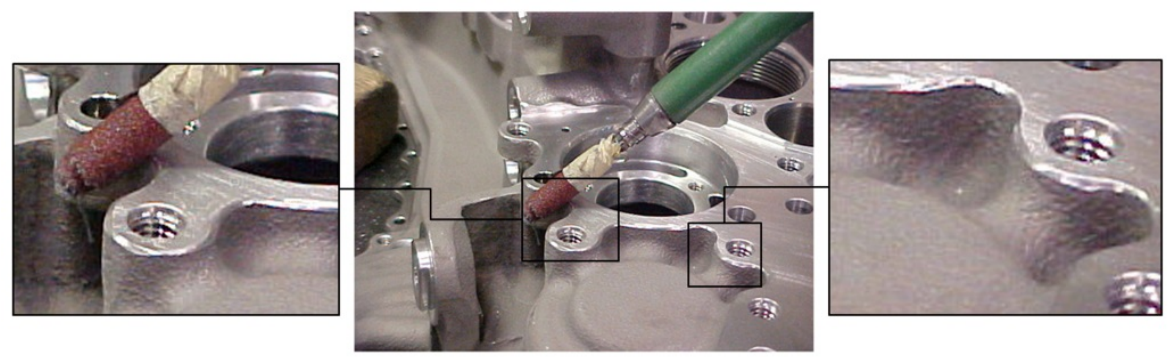

Fig. 8. Manual finishing of the housing (center) and details of the machined edges (left and right)

Acknowledgments. The authors want to express their gratitude to Luciano Passoni, Davide Passoni and Lino Ferrari from SIR S.p.A. (Modena, Italy), for their technical and managerial contribution to the project, and AVIO S.p.A. (Torino, Italy) for supporting the experimental tests.

\section{References}

1. Jayaweera, N., Webb, P.: Robotic Edge Profiling of Complex Components. Industrial Robot: An International Journal 38, 38-47 (2010)

2. ElMaraghy, H.A.: Flexible and Reconfigurable Manufacturing Systems Paradigms. International Journal of Flexible Manufacturing Systems 17, 261-276 (2005)

3. Bi, Z.M., Lang, S.Y.T.: General Modular Robot Architecture and Configuration Design. In: 2005 IEEE International Conference on Mechatronics and Automation, pp. 268-273. IEEE Press, New York (2001)

4. Pellicciari, M., Leali, F., Andrisano, A.O., Pini, F.: Enhancing Changeability of Automotive Hybrid Reconfigurable Systems in Digital Environments. International Journal on Interactive Design and Manufacturing 6, 251-263 (2012)

5. Andrisano, A.O., Leali, F., Pellicciari, M., Pini, F., Vergnano, A.: Hybrid Reconfigurable System Design and Optimization Through Virtual Prototyping and Digital Manufacturing Tools. International Journal on Interactive Design and Manufacturing 6, 17-27 (2012)

6. Pan, Z., Zhang, H.: Robotic machining from a programming to a process control: a complete solution by force control. Industrial Robot: An International Journal 35, 400-409 (2008)

7. DePree, J., Gesswein, C.: Robotics Machining White Paper Project. Halcyon Development - Robotic Industries Association (RIA)

8. Abderrahim, M., Khamis, A., Garrido, S., Moreno, L.: Accuracy and Calibration Issues of Industrial Manipulators. In: Huat, L.K. (ed.) Industrial Robotics: Programming, Simulation and Application, pp. 131-146. Pro Literatur Verlag, Germany (2006)

9. Shiakolas, P.S., Conrad, K.L., Yih, T.C.: On the Accuracy, Repeatability, and Degree of Influence of Kinematics Parameters for Industrial Robots. International Journal of Modelling and Simulation 22, 1-10 (2002)

10. Pan, Z., Zhang, H., Zhu, Z., Wang, J.: Chatter Analysis of Robotic Machining Process. Journal of Material Processing Technology 173, 301-309 (2006)

11. Cherif, M., Knevez, J.Y., Ballu, A.: Thermal Aspects on Robot Machining Accuracy. In: Proceedings of IDMME - Virtual Concep. (2010) 
12. Pan, Z., Polden, J., Larkin, N., Van Duin, S., Norrish, J.: Recent Progress on Programming Methods for Industrial Robots. Robotics and Computer-Integrated Manufacturing 28, 87-94 (2012)

13. COMET Project - Plug-and-Produce COmponents and METhods for Adaptive Control of Industrial Robots Enabling Cost Effective, High Precision Manufacturing in Factories of the Future. In: European 7th Framework Programme, reference number 258769

14. Li, X., Zhang, B.: Toward General Industrial Robot Cell Calibration. In: 5th IEEE International Conference on Robotics, Automation and Mechatronics (RAM), pp. 137-142. IEEE Press, New York (2011)

15. Lu, T., Lin, G.C.I.: An On-Line Relative Position and Orientation Error Calibration Methodology for Workcell Robot Operations. Robotics and Computer-Integrated Manufacturing 13, 89-99 (1997)

16. Lim, H.K., Kim, D.H., Kim, S.R., Kang, H.J.: A Practical Approach to Enhance Positioning Accuracy for Industrial Robots. In: ICCAS-SICE 2009 ICROS-SICE International Joint Conference 2009, pp. 2268-2273. IEEE Press, New York (2009)

17. Mooring, B.W., Roth, Z.S., Driels, M.R.: Fundamentals of Manipulator Calibration. John Wiley \& Sons, Inc., New York (1991)

18. Elatta, A.Y., Li, P.G., Fan, L.Z., Yu, D.Y.: An Overview of Robot Calibration. Information Technology Journal 3, 74-78 (2004)

19. Mustafa, S.K., Pey, Y.T., Guilin, Y., I-Ming, C.: A geometrical Approach for Online Error Compensation of Industrial Manipulators. In: IEEE/ASME 2010 International Conference on Advance Intelligent Mechatronics, pp. 738-743. IEEE Press, New York (2010)

20. Gan, Z., Sun, Y., Tang, Q.: In-Process Relative Robot Workcell Calibration. US Patent 6,812,665 (November 2, 2004)

21. Cheng, F.S.: The Method of Recovering Robot TCP Positions in Industrial Robot Application Programs. In: 2007 IEEE International Conference on Mechatronics and Automation (ICMA 2007), pp. 805-810. IEEE Press, New York (2007)

22. Bley, H., Bernerdi, M., Franke, C., Seel, U.: Process-based assembly planning using a simulation system with cell calibration. In: 2001 IEEE International Symposium on Assembly and Task Planning, pp. 116-121. IEEE Press, New York (2001)

23. Ribeiro, F., McMaster, R.: A Low Cost Cell Calibration Technique and its PC based Control Software. In: 1997 IEEE International Symposium on Industrial Electronics (ISIE 1997), vol. 3, pp. 840-845. IEEE Press, New York (1997)

24. Andrisano, A.O., Leali, F., Pellicciari, M., Vergnano, A.: Engineering Method for Adaptive Manufacturing Systems Design. International Journal on Interactive Design and Manufacturing 3, 81-91 (2009)

25. ABB Product Specification Articulated Robot IRB 2400 - 3HAC9112-1, Rev. T 